leads to genome-wide changes in chromatin structure and transcription factor binding. Leukemia. 2012;26:1829-41.

6. Sun XJ, Wang Z, Wang L, Jiang Y, Kost N, Soong TD, et al. A stable transcription factor complex nucleated by oligomeric AML1-ETO controls leukaemogenesis. Nature. 2013;500:93-7.

7. Marcotte EM, Tsechansky M. Disorder, promiscuity, and toxic partnerships. Cell. 2009;138:16-8.

8. Vavouri T, Semple JI, Garcia-Verdugo R, Lehner B. Intrinsic protein disorder and interaction promiscuity are widely associated with dosage sensitivity. Cell. 2009;138:198-208.

9. Rosenbauer F, Tenen DG. Transcription factors in myeloid development: balancing differentiation with transformation. Nat Rev Immunol. 2007;7:105-17.

10. Semple JI, Vavouri T, Lehner B. A simple principle concerning the robustness of protein complex activity to changes in gene expression. BMC Syst Biol. 2008;2:1.

11. Pabst T, Mueller BU, Harakawa N, Schoch C, Haferlach T, Behre $\mathrm{G}$, et al. AML1-ETO downregulates the granulocytic differentiation factor C/EBPalpha in $\mathrm{t}(8 ; 21)$ myeloid leukemia. Nat Med. 2001;7:444-51.
12. Westendorf JJ, Yamamoto CM, Lenny N, Downing JR, Selsted ME, Hiebert SW. The $\mathrm{t}(8 ; 21)$ fusion product, AML-1-ETO, associates with $\mathrm{C} / \mathrm{EBP}$-alpha, inhibits $\mathrm{C} / \mathrm{EBP}$-alpha-dependent transcription, and blocks granulocytic differentiation. Mol Cell Biol. 1998;18:322-33.

13. Chan WY, Follows GA, Lacaud G, Pimanda JE, Landry JR, Kinston S, et al. The paralogous hematopoietic regulators Lyl1 and $\mathrm{Scl}$ are coregulated by Ets and GATA factors, but Lyl1 cannot rescue the early Scl-/- phenotype. Blood. 2007;109:1908-16.

14. Ohlsson E, Schuster MB, Hasemann M, Porse BT. The multifaceted functions of C/EBPalpha in normal and malignant haematopoiesis. Leukemia. 2016;30:767-75.

15. Ptasinska A, Assi SA, Martinez-Soria N, Imperato MR, Piper J, Cauchy $\mathrm{P}$, et al. Identification of a dynamic core transcriptional network in $\mathrm{t}(8 ; 21)$ AML that regulates differentiation block and self-renewal. Cell Rep. 2014;8:1974-88.

16. Loke J, Chin PS, Keane P, Pickin A, Assi SA, Ptasinska A, et al. $\mathrm{C} / \mathrm{EBPalpha}$ overrides epigenetic reprogramming by oncogenic transcription factors in acute myeloid leukemia. Blood Adv. 2018;2:271-84.

\title{
GATA1 epigenetic deregulation contributes to the development of AML with NPM1 and FLT3-ITD cooperating mutations
}

\author{
Paolo Sportoletti $\mathbb{I I}^{1}$ - Letizia Celani ${ }^{1} \cdot$ Emanuela Varasano $^{1} \cdot$ Roberta Rossi $^{1} \cdot$ Daniele Sorcini $^{1} \cdot$ Chiara Rompietti $^{1}$. \\ Francesca Strozzini ${ }^{1}$ - Beatrice Del Papa ${ }^{1}$ - Valerio Guarente ${ }^{1}$ - Giulio Spinozzi ${ }^{1}$ - Debora Cecchini ${ }^{1}$ • \\ Oxana Bereshchenko ${ }^{2} \cdot$ Torsten Haferlach $^{3} \cdot$ Maria Paola Martelli $^{1} \cdot$ Franca Falzetti $^{1} \cdot$ Brunangelo Falini $^{1}$
}

Supplementary information The online version of this article (https:// doi.org/10.1038/s41375-019-0399-7) contains supplementary material, which is available to authorized users.

Paolo Sportoletti

paolo.sportoletti@unipg.it

$\bowtie$ Brunangelo Falini

brunangelo.falini@unipg.it

1 Centro di Ricerca Emato-Oncologica (CREO), University of Perugia, Perugia 06132, Italy

2 Section of Pharmacology, Toxicology and Chemotherapy, University of Perugia, Perugia 06132, Italy

3 MLL Munich Leukemia Laboratory, Munich 81377, Germany

\section{To the Editor:}

About 20 recurrently mutated genes are known to be involved in the molecular pathogenesis of acute myeloid leukemia (AML) [1]. Among them, Nucleophosmin (NPM1) and FLT3-ITD mutations frequently occur together in adults with AML [2], suggesting cooperative leukemogenesis. To date, the molecular consequences of these cooperative genetic alterations in AML are still elusive.

To address this issue, we crossed Npml and Flt3-ITD mutant mice demonstrating the onset of lethal AML (Fig. 1a and Figure S1). Interestingly, the cumulative mutant allele burden influenced the leukemic phenotype, penetrance and latency. NPMI/Flt3-ITD double heterozygous mice $\left(\mathrm{Npml}^{+/ \mathrm{TCTG}} ; \mathrm{Flt3}^{+/ \mathrm{ITD}}\right)$ displayed a significantly reduced overall survival compared to single mutant or wild-type mice. Survival was further reduced in mice with two NPM1 mutant alleles and one Flt3-ITD allele $\left(\mathrm{Npml}^{\mathrm{TCTG} / \mathrm{TCTG}} ; \mathrm{Flt}^{+/ \mathrm{ITD}}\right)$. 
A
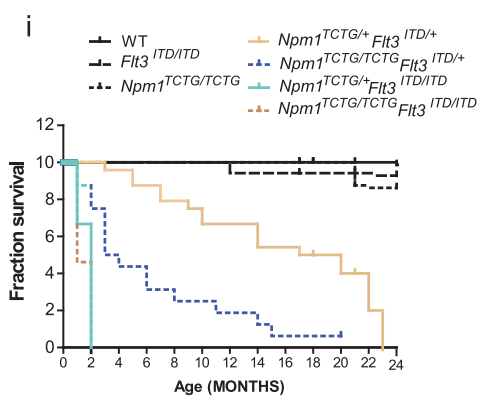
Age (MONTHS)

B

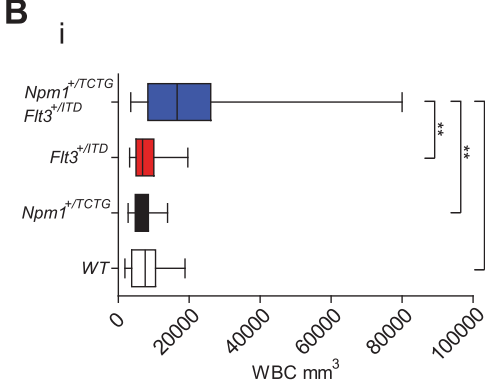

V

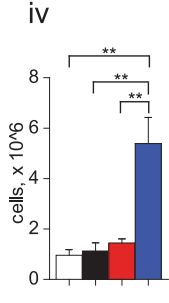

GMP

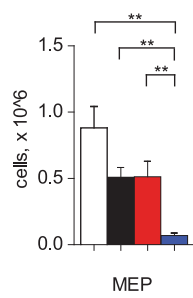

$\mathrm{C}_{\mathrm{i}}$

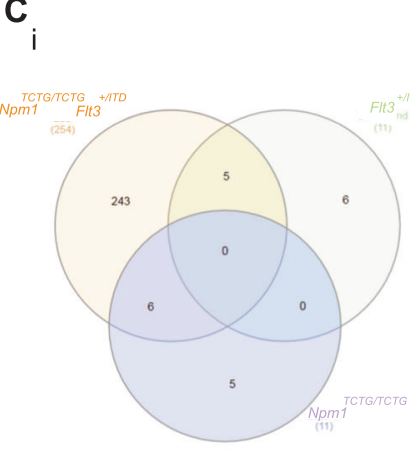

ii

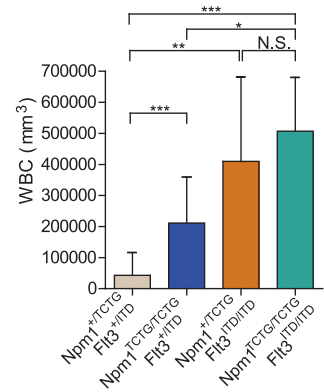

iii
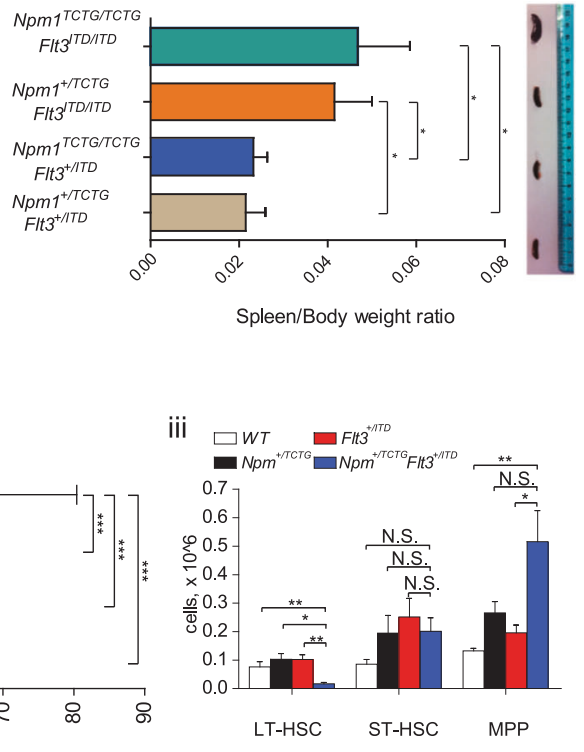

$\mathrm{fL}$
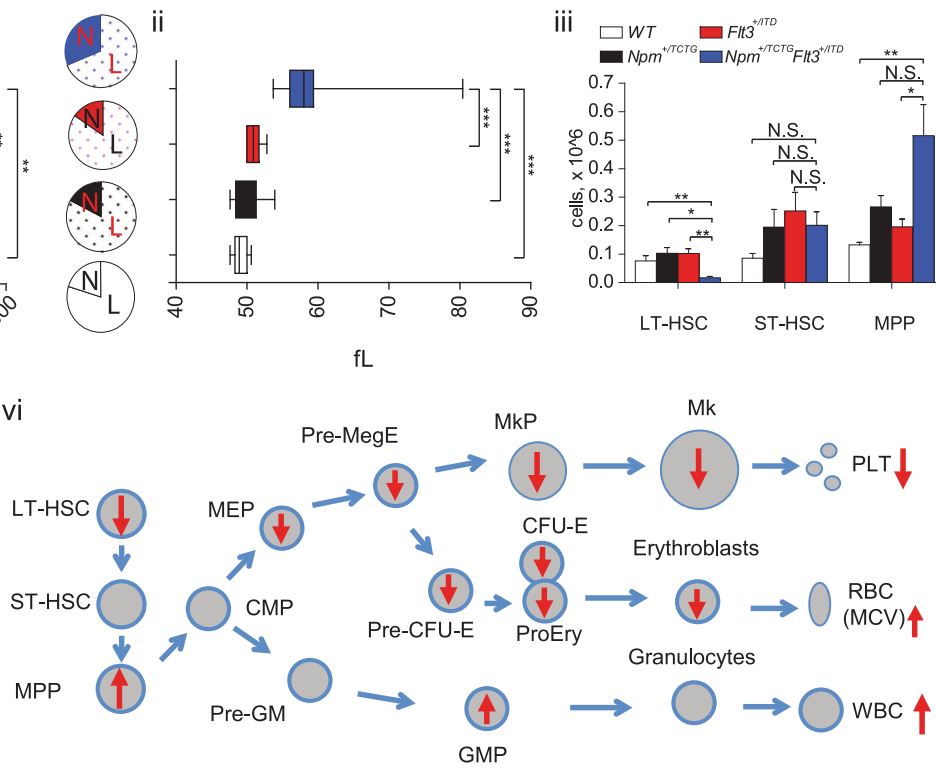

D
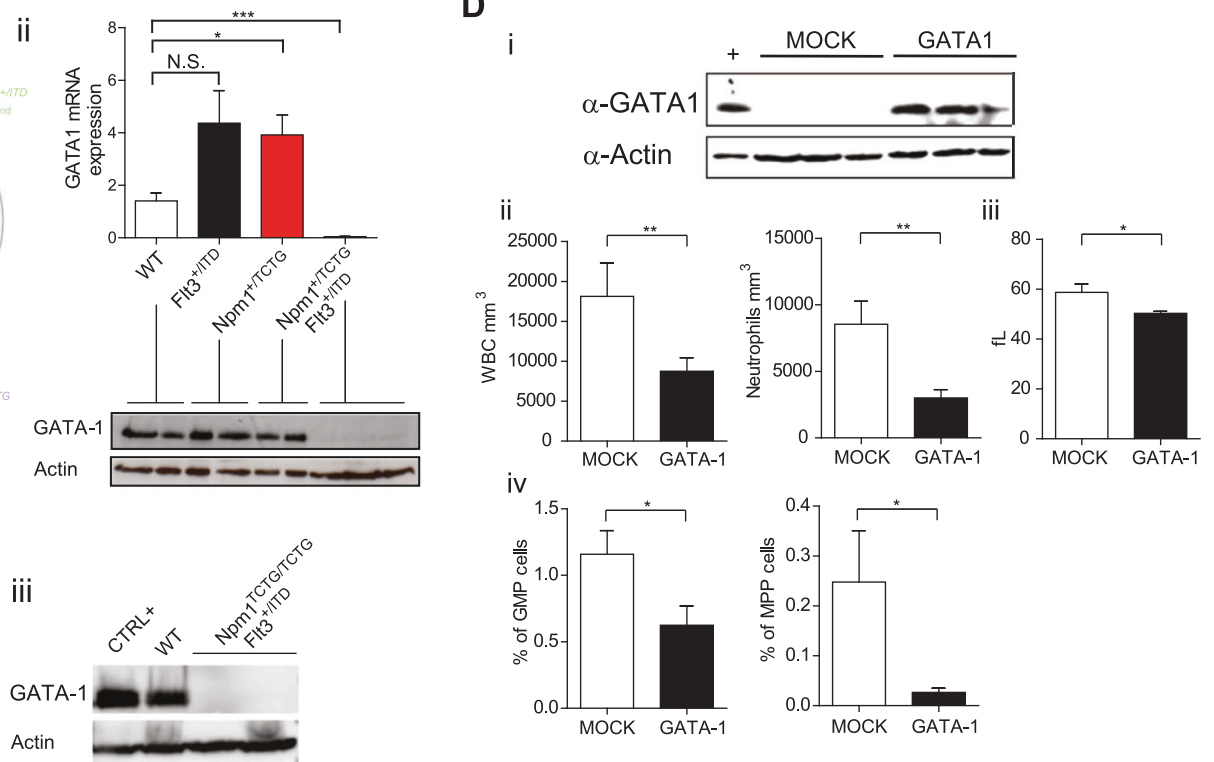

Interestingly, Flt3-ITD homozygous mice $\left(\mathrm{Npml}^{+/ \mathrm{TCTG}}\right.$; Flt $3^{\mathrm{ITD} / \mathrm{TD}}$ and $\mathrm{Npm} 1^{\mathrm{TCTG} / \mathrm{TCTG}} ; \mathrm{Flt}^{\mathrm{ITD} / \mathrm{TD}}$ ) showed the higher white blood cell (WBC) counts, a significant spleen enlargement (Fig. 1a ii-iii) and developed AML rapidly regardless of NPM1 mutation dosage, suggesting that in the context of a high FLT3 kinase activity even small NPM1 
Fig. 1 Lethal acute myeloid leukemia (AML) in Npm1/Flt3-ITD mice is preceded by changes in myeloid and erythroid cells associated with GATA1 deregulation. a (i) Kaplan-Meier plot of mouse survival according to the indicated genotypes $(n=8$ to 24 per genotype); $\mathrm{Npm}^{+/ \mathrm{TCTG}} ; \mathrm{Flt3}^{+/ \mathrm{TD}}$ mice display a median survival of 18.5 months versus 21 months of $N p m 1^{+/ \text {TCTG }}$ or Flt $3^{+/ I T D}$ mice or 22.5 months of wild-type controls $(p<0.0001$, logrank test). (ii) Changes in white blood cell (WBC) counts of Npm1 $1^{+T C T G} ;$ Flt $3^{\text {ITD/ITD }}$ $(n=3), \mathrm{Npm}^{\text {TCTG/TCTG}} ; \mathrm{Flt3}^{\text {ITD/ITD }}(n=3), \mathrm{Npm}^{+/ T C T G} ; \mathrm{Flt}^{+/ I T D}(n$ $=6)$ and $N p m 1^{T C T G / T C T G} ; F l t 3^{+/ T T D}(n=9)$ mice. (iii) Spleen weight to total body weight ratio in the indicated genotypes. Spleen ratio in $\mathrm{Npm}^{+/ T C T G} ; \mathrm{Flt3}^{I T D / I T D}(n=3)$ and Npm1 $1^{\text {TCTG/TCTG }} ;$ Flt $^{I T D / I T D}(n=4)$ mice was two fold greater than in $N p m 1^{+/ T C T G} ; F l t 3^{+I T D}(n=5)$ and $\mathrm{Npm1}^{\text {TCTG/TCTG }} ;$ Flt $^{+/ I T D}(n=18)$ leukemic mice $(0.041 \pm 0.014$ and $0.046 \pm 0.023$ vs $0.017 \pm 0.012$ and $0.025 \pm 0.016 p<0.001$ by oneway analysis of variance (ANOVA) analysis). b (i) Significant differences in WBC count in Npm1 $1^{+/ T C T G} ;$ Flt $3^{+/ I T D}$ compared to Npm1 ${ }^{+/ T C T G} ; \mathrm{Flt}^{+/+}, \mathrm{Npm}^{+/+} ; \mathrm{Flt}^{+/ I T D}$ and $\mathrm{Npm}^{+/+} ; \mathrm{Flt}^{+/+}$littermate groups ( $n=12$ to 20 per genotype); pie charts show neutrophils $(\mathrm{N})$ and lymphocytes (L) percentages. (ii) Mean corpuscolar volume (MCV) values in preleukemic mice ( $n=12$ to 20 per genotype). (iii-v) Flow-cytometric analysis of bone marrow stem and progenitor cell compartment sizes, including long-term hematopoietic stem cells (LTHSCs; lin $^{-}$Sca- $1^{+}$c-kit ${ }^{+}$CD $34^{-}{ }^{-}{ }^{-1} 3^{-}$), short-term HSCs (ST-HSCs; lin $\mathrm{Sca}-1^{+} \mathrm{c}-\mathrm{kit}^{+} \mathrm{CD} 34^{+} \mathrm{Flt} 3^{-}$), multipotent progenitors (MPPs; lin ${ }^{-} \mathrm{Sca}-1^{+} \mathrm{c}-\mathrm{kit}{ }^{+} \mathrm{CD} 34^{+} \mathrm{Flt} 3^{+}$), granulocyte/monocyte progenitors (GMPs $\mathrm{Lin}^{-} \mathrm{Sca}-1^{-} \mathrm{cKit}^{+} \mathrm{CD} 34^{+} \mathrm{Fc} \gamma \mathrm{RII} / \mathrm{III}^{\mathrm{hi}}$ ) and common megakaryocyte-erythroid progenitor (MEP; $\mathrm{Lin}^{-} \mathrm{Sca}-1^{-} \mathrm{cKit}^{+} \mathrm{CD} 34$ ${ }^{-}$Fc $\gamma$ RII/IIII ${ }^{\mathrm{lo}}$ ) populations ( $n=4$ to 10 per genotype). (vi) Summary of hemopoietic changes in $N p m 1^{+/ T C T G} ; F l t 3^{+/ I T D}$ mice. c (i) Overlap of differently gene expression profiling (GEP) of $\mathrm{Npm}^{\text {TCTG/TCTG }} ; \mathrm{Flt}^{+/+}$, $\mathrm{Npm}^{+/+} ; \mathrm{Flt3}^{+/ I T D}$ and Npm1 ${ }^{\text {TCTG/TCTG }} ; \mathrm{Flt3}^{+/ I T D}$ compared to Npm1 ${ }^{+/+} ; F l t 3^{+/+}$( $n=3$ mice for each genotype). (ii) GATA1 messenger RNA (mRNA) and protein expression in the bone marrow (BM) of the indicated genotypes. (iii) GATA1 protein expression in lineagedepleted BM cells from the indicated genotypes. d (i) Enforced expression of GATA1 protein in the BM of mice transplanted with $\mathrm{Npm}^{+/ T C T G}$;Flt ${ }^{+/ I T D}$ LSK ( $n=4$ to 12 ) infected with an inducible GATA1 lentiviral system and killed 2 months after transplantation. (ii, iii) Significant differences in WBC counts, neutrophils and MCV values in the peripheral blood (PB) of GATA1-rescued mice. (iv) Frequency of MPP and GMP populations in MOCK $(n=10)$ and GATA1 $(n=16)$ infected mice. Data represent the mean \pm SD. N.S. not significant; $* p<0.05, * * p<0.01, * * * p<0.001$; unpaired $t$-test with Welch's correction

mutant levels are leukemogenic. This supports a direct oncogenic effect of the NPM1 mutant, beside the haploinsufficient tumor suppressor effects of the concomitant loss of one NPM1 allele [3,4]. Additionally, our data are in line with the clinical observation that normal karyotype AML with high FLT3-ITD levels have a poor outcome [5].

The analysis of the $N p m 1^{+/ T C T G} ; F_{l t 3}{ }^{+/ I T D}$ genotype, which is characterized by a longer AML latency, demonstrated changes in the myeloid and erythroid cells before leukemia onset. WBC counts and mean corpuscular volume (MCV) were significantly higher in $N p m 1^{+/ T C T G} ; F_{l t 3}{ }^{+/ I T D}$ mice than in wild-type, $\mathrm{Npml}^{+/ \mathrm{TCTG}}$ and $\mathrm{Flt}^{+/ / \mathrm{TD}}$ groups (Fig. $1 \mathrm{~b}$ i-ii). Flow cytometry analysis of bone marrow (BM) populations showed that leukocytosis was associated to reduced long-term hematopoietic stem cells (HSCs), significant expansion of multipotent progenitor (MPP) cells and a 6.1-fold increase of granulocyte/monocyte progenitors (GMPs) (Fig. 1b iii-iv). $\mathrm{NpmI}^{+/ T C T G} ; \mathrm{Flt}^{+/ I T D}$ mice had decreased number of immature and recirculating B-cell BM populations (Figure S2). Erythrocyte changes reflected a significant reduction in the corresponding BM populations at different differentiation stages including myelo-erythroid progenitors (MEP), premegakaryocyte-erythrocyte progenitors (PreMegE), precolony forming unit-erythroid (pre-CFU-E), CFU-E and proerythroblasts (proEry) that resulted almost absent (Fig. 1b v-vi and Figure S3). In physiological hematopoiesis, FLT3 upregulation is important in sustaining MPP and GMP but not MEP potential [6]. In $\mathrm{NpmI}^{+/ T C T G} ; \mathrm{Flt3}^{+/ I T D}$ mice, constitutive Flt3-ITD signaling boosts the myeloid bias and influences the megakaryocyte/erythroid lineage fates, strongly suggesting the capacity of the NPM1 mutant to synergize with a FLT3 activity. These findings appear to define the cellular background for the acquisition of additional events for AML onset.

Comparative gene expression profiling (GEP) studies on total BM revealed a large number of differentially expressed genes in $\mathrm{NpmI}^{\text {TCTG/TCTG}} ; \mathrm{Flt3}^{+/ I T D}$ leukemic mice compared to $F l t 3^{+/ I T D}, N p m 1^{T C T G / T C T G}$ and wild-type groups. A total of 254 genes were differentially expressed in Npm1 ${ }^{\text {TCTG/TCTG }}$;Flt $3^{+/ I T D}$ mice compared to wild-type littermates (42 up-regulated; 214 down regulated) (Table S1). Interestingly, when compared with wild-type, there were 243 transcripts whose expression was changed only in Npm1 $1^{\text {TCTG/TCTG }} ;$ Flt $^{+/ I T D}$ cells (Fig. 1c i). There were no transcripts commonly altered in all pairwise comparisons. Hoxa9 scored as one of the most up-regulated genes in $N p m 1^{T C T G / T C T G} ; F l t 3^{+/ I T D}$ mice, a characteristic hallmark of NPM1-driven leukemia. Similar findings were present in mice with early-stage AML.

Pathway analysis showed different changes when comparing $N p m 1^{\text {TCTG/TCTG }} ; \mathrm{Flt}^{+/ I T D}$ to wild-type mice. Among these, we found pathways involved in hematopoietic cell lineage development, the B-cell receptor signaling and the immunoregulatory interactions between lymphoid and nonlymphoid cells. Additionally, Npm1 $1^{\text {TCTG/TCTG }} ; F_{l t} 3^{+/ I T D} \mathrm{BM}$ samples displayed a significant deregulation of factors involved in megakaryocyte development and platelet production. Interestingly, several genes associated with this pathway were linked to a GATA transcriptional signature, including GATA1, Zpfm1, Rac1 and Ehd2. The latter showed a significant downregulation, with GATA1 displaying the lower levels (Figure S4). A similar expression signature was present in lineage-depleted BM cells used to exclude biases related to the different cellular composition of leukemic versus wild-type mice (Figure S5). In this context, we found a higher number of deregulated GATA gene family members including GATA1, GATA2 and GATA3.

Results of GEPs and the presence of alterations in erythropoiesis before AML development prompted us to focus 
A
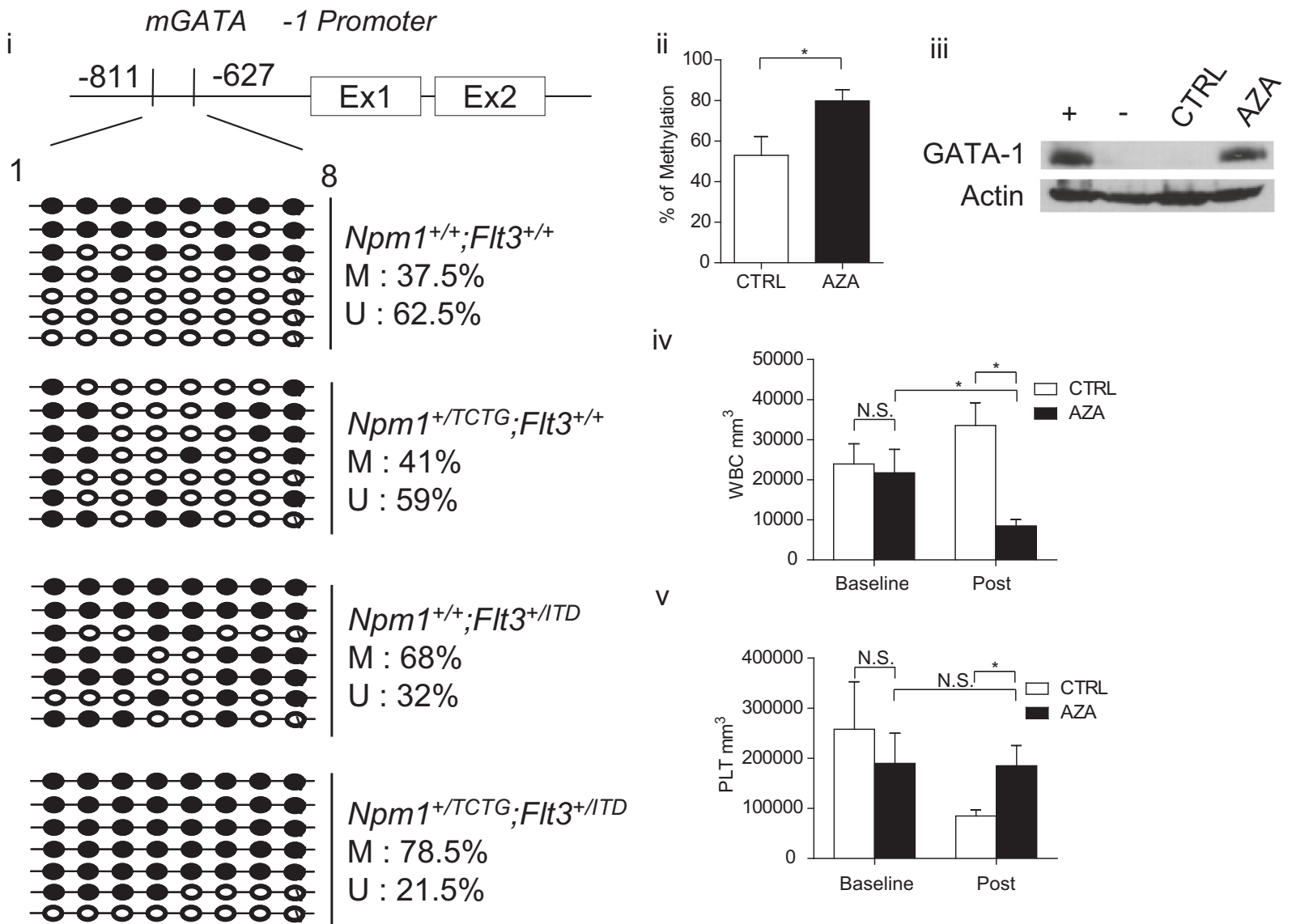

Npm1 1/TCTG;Flt3 ${ }^{+/ I T D}$

$\mathrm{M}: 78.5 \%$

$U: 21.5 \%$

iv

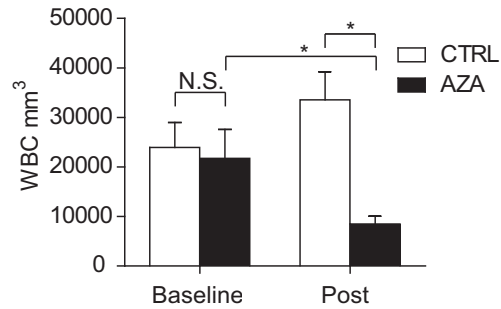

V

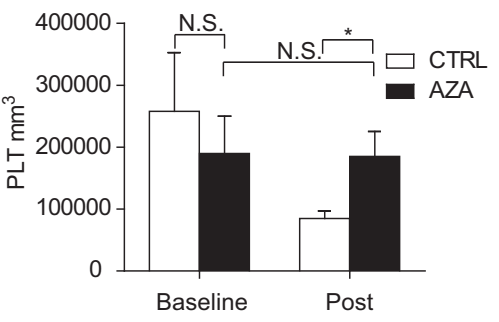

$\mathrm{vi}$

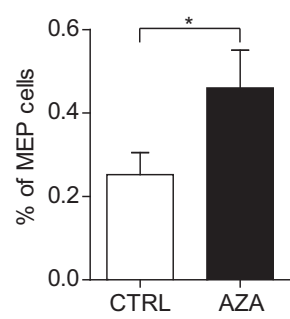

B

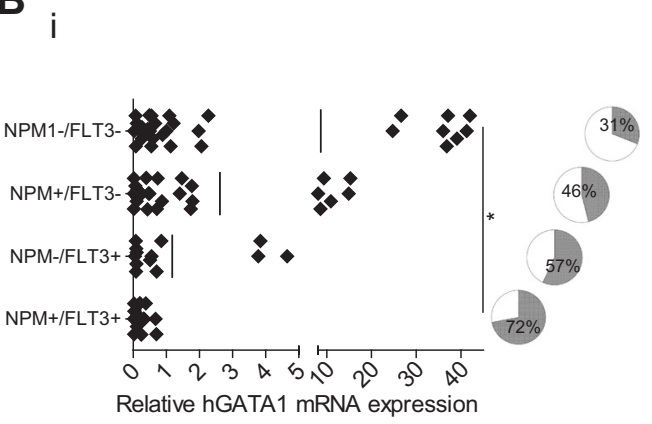

vii

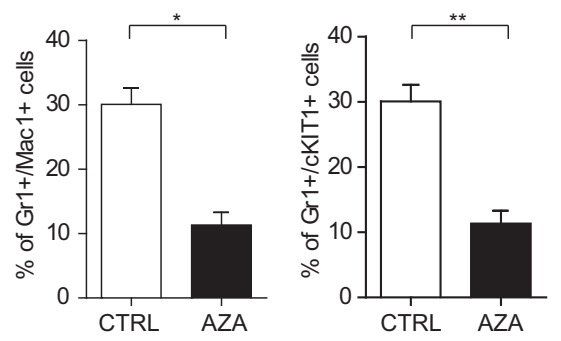

ii

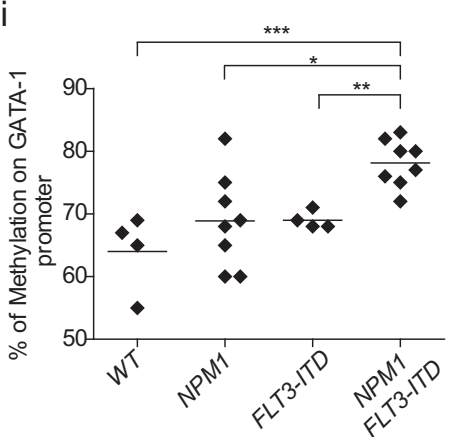

iii

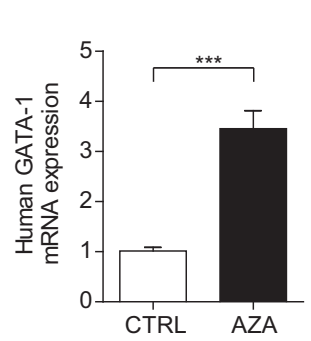

on GATA1, the master regulator of erythroid differentiation. Notably, BM changes in $\mathrm{Npml}^{+/ \mathrm{TCTG}} ; \mathrm{Flt}^{+/ / \mathrm{TD}}$ mice were accompanied by a dramatic reduction of GATA1 messenger
RNA (mRNA) and complete loss and substantial downregulation of protein expression in both total or lineagedepleted BM (Fig. 1c ii-iii). The extent of GATA1 
Fig. 2 Decreased GATA1 expression levels in human and mouse NPM1/FLT3-ITD mutated acute myeloid leukemia (AML) depends on promoter methylation. a (i) Analysis of DNA methylation at the mouse GATA1 locus by sequencing of PCR clones derived from sodium bisulfite-treated mouse genomic DNA extracted from the bone marrow (BM). Each row of circles represents the sequence of an individual clone; open circles indicate unmethylated $\mathrm{CpG}$ sites and closed circles indicate methylated $\mathrm{CpG}$ sites. (ii) Methylation status of the GATA1 promoter as determined by the Methylight assay. (iii) GATA1 protein expression in the $\mathrm{BM}$ of Aza-treated mice $(n=5)$. (iv, v) Changes in white blood cell (WBC) and platelet (PLT) counts of $N p m 1^{+I T C T G} ;$ Flt $3^{+/ I T D}$ mice treated with Aza $(n=5$ to 10 per treatment group). (vi) Frequencies of MEP and Ter119 cells in the BM of Azatreated mice ( $n=5$ to 12 per treatment group). (vii) Frequencies of $\mathrm{Gr} 1+/ \mathrm{cKit}+$ immature and $\mathrm{Gr} 1+\mathrm{Mac} 1+$ mature myeloid cells in the spleen of untreated vs 5-Aza-treated leukemic mice $(n=5)$. b (i) GATA1 mRNA average expression in AML patients with NPM1/ FLT3-ITD mutation compared to unmutated, NPM1 and FLT3-ITD single mutant ( $p<0.05$ comparing all the groups); pie charts indicate the percentage of patients with GATA1 expression below the median in the indicated mutation group. (ii) GATA1 promoter methylation frequency in AML patients with NPM1/FLT3-ITD mutation $(n=8)$ as compared to unmutated $(n=4)$, NPM1 $(n=8)$ and FLT3-ITD $(n=4)$ single mutant. (iii) GATA1 mRNA levels in the BM of human AML patients $(n=3)$ before and after in vivo Aza treatment. N.S. not significant; $* p<0.05, * * p<0.01 ; * * * p<0.001$; unpaired $t$-test with Welch's correction

deregulation correlated with the degree of the myeloid phenotypic changes (Figure S6). In vivo restoration of GATA1 expression in $\mathrm{Npm}^{+/ \mathrm{TCTG}_{j}} \mathrm{Flt3}^{+/ \mathrm{ITD}} \mathrm{Lin}^{-} \mathrm{Sca}-1$ ${ }^{+} \mathrm{CKit}^{+}$cells using a conditional lentiviral system (Fig. 1d i and Figure S7A) rescued most of the preleukemic phenotype which included a significant reduction of WBC and neutrophils in peripheral blood (PB) and a decrease in percentage of MPP and GMP (Fig. 1d ii,iv and Figure S7B). Interestingly, GATA1 re-expression also led to a rescue of macrocytosis with a significant reduction of the MCV values from 58.7 to $50.2 \mathrm{fL}$ (Fig. 1d iii). Moreover, spleens from GATA1-rescued mice showed a decrease in size and a reduction of myeloid-infiltrating cells compared to controls (Figure S7C). Collectively, these findings provide evidence that deregulation of GATA1 plays a key role in the hemopoietic changes preceding AML in $\mathrm{Npml}^{+/ \mathrm{TCTG}} ; \mathrm{Flt}^{+/ / \mathrm{TD}}$ mice. This is consistent with the concept that in blood cell precursors, GATA1 is necessary for erythroid lineage differentiation and antagonizes the activity of myeloid transcription factors [7].

Our findings are consistent with the observation that GATA1 heterozygous knock-out female mice frequently develop a myeloproliferative disorder with a splenic accumulation of proerythroblasts and megakaryocytes, anemia and thrombocytopenia [7]. Moreover, recurrent GATA1 mutations abrogating the expression of the full-length GATA1 have been found in myeloid proliferations related to Down syndrome, including transient abnormal myelopoiesis and megakaryoblastic AMLs [8]. Interestingly, FLT3-ITD mutations were more frequent in AML patients who lacked GATA1 expression [9] and even $I D H$-mutated AML patients displayed a distinct methylation signature, including the aberrant hypermethylation of GATA1/2 gene promoter [10].

Proteasome inhibition of $\mathrm{Npml}^{+/ \mathrm{TCTG}} ; \mathrm{Flt3}^{+/ / \mathrm{TD}} \mathrm{BM}$ cells in vitro did not rescue GATA1 protein expression (Figure S8A), suggesting that NPMI and Flt3-ITD mutations regulate GATA1 transcription. Thus, we explored changes in the methylation status of the GATA1 promoter region (from -811 to $-627 \mathrm{bp}$ ) and observed dense DNA methylation in $\mathrm{NpmI}^{+/ \mathrm{TCTG}} ; \mathrm{Flt3}^{+/ / \mathrm{TD}}$ samples (Fig. 2a i-ii). To support GATA1 epigenetic silencing as a mechanism favoring AML, we treated $\mathrm{Npm}^{+/ \mathrm{TCTG}} ; \mathrm{Flt}^{+/ / \mathrm{ITD}}$ mice with the DNA methyltransferase inhibitor 5-aza-deoxycytidine (5-Aza-dC). This resulted in the reactivation of GATA1 expression in BM (Fig. 2a iii and Figure S8B), normalization of leukocytosis and prevention of a drop in platelet counts (Fig. 2a iv-v). Although 5-Aza-dC treatment had no impact on MCV, both MEP and Ter119 cells were significantly expanded in treated animals (Fig. 2a vi). Flow cytometry of spleen demonstrated a significant reduction of both mature and immature myeloid cells in 5-Aza-dCtreated mice (Fig. 2a vii). Our findings are reminiscent of the differential methylation of GATA target genes previously reported in AML mouse models combining FLT3ITD to either IDH mutants [11] or TET2 loss [12]. The higher 5'-GATA1 methylation in $\mathrm{Npml}^{+/ T C T G} ; \mathrm{Flt}^{+/ I T D}$ mice points to a gene dose effect for GATA1 during leukemogenesis being finely tuned by $\mathrm{CpG}$ methylation. This suggests that NPM1 alterations may contribute to epigenetic modifications, especially in the presence of other mutations, such as Flt3-ITD. This view is consistent with NPM1 being an histone chaperone that interacts with linker histone H1, plays a role in sperm chromatin remodeling, enhances acetylation-dependent chromatin transcription and controls ribosomal DNA gene transcription [13].

To assess the relevance of mouse findings to human AML, we correlated GATA1 mRNA expression with the NPM1 and FLT3-ITD mutational status in the BM of 47 AML, demonstrating that patients harboring both mutations displayed the lowest expression of GATA1 (Fig. 2b i). The median GATA1 level of 0.44 was arbitrarily used as cut-off to distinguish high and low expressing patients. AMLs with low GATA1 were more frequent among NPM1-mutated/ FLT3-ITD AMLs than unmutated, single NPM1 or FLT3ITD-mutated patients (72 vs 31,46 and $57 \%$ respectively; pie charts in Fig. $2 b$ i). These findings were further validated in an independent database of 266 AML of the Munich Leukemia Laboratory (www.ncbi.nlm.nih.gov/geo, accession number (GSE16015) (Figure S9). Additionally, we explored the methylation status of the GATA1 promoter region in 24 patients, revealing a significant DNA methylation in NPM1-mutated/FLT3-ITD samples with an 
average of $78.1 \% \pm 1.3$ methylated $\mathrm{CpG}$ sites compared to $68.8 \% \pm 2.6$ in NPM1-mutated only, $69 \% \pm 0.7$ in FLT3ITD-mutated only and $64 \% \pm 3.1$ in wild-type (Fig. $2 \mathrm{~b}$ ii). Finally, the analysis of GATA1 expression levels was performed in BM samples from 3 NPM1-mutated/FLT3ITD patients treated with 5 -Aza-dC, revealing a significant up-regulation of GATA1 mRNA after the first cycle (Fig. 2b iii). These data corroborate a potential role for DNA methylation of GATA1 promoter in the development of NPM1-mutated/FLT3-ITD AML. Our findings are also of potential clinical relevance, as GATA1 transcriptional response to 5-Aza-dC in mice results in significant improvement of the myeloid phenotype. Similarly, we observed GATA1 mRNA up-regulation in two NPM1mutated/FLT3-ITD AML patients upon 5-Aza-dC treatment.

In conclusion, we identified deregulation of GATA1 as a new feature of Npml/Flt3-ITD AML in mice and humans. This is an early event altering the HSC fate and sensitizing cells to further malignant transformation. Our model may also be valuable for further assessment of FLT3 inhibitors [14] and other drugs that have been shown to be active against NPMI-mutated AML [15].

Acknowledgements This work was supported by AIRC IG 2016 no. 18568 (to BF), ERC Adv Grant 2016 no. 740230 (to BF) and ERC Cons Grant 2016 no. 725725 (to MPM).

Author contributions PS and BF conceived the study. LC, EV, RR, DS, CR, FS, BDP, CR and VG performed the experiments and analyzed the data. DS, DC, OB and LC performed cytometric analysis. LC, DS, RR and VG performed molecular analysis. EV, RR, FS and LC carried out histological analysis. PS and GS constructed analytical and visualization tools and databases. TH, provided samples. MPM and FF provided logistical support. PS and BF wrote the manuscript. All authors approved the manuscript.

\section{Compliance with ethical standards}

Conflict of interest BF applied for a patent on the clinical use of NPM1 mutants. The other authors declare that they have no conflict of interest.

Publisher's note: Springer Nature remains neutral with regard to jurisdictional claims in published maps and institutional affiliations.

Open Access This article is licensed under a Creative Commons Attribution 4.0 International License, which permits use, sharing, adaptation, distribution and reproduction in any medium or format, as long as you give appropriate credit to the original author(s) and the source, provide a link to the Creative Commons license, and indicate if changes were made. The images or other third party material in this article are included in the article's Creative Commons license, unless indicated otherwise in a credit line to the material. If material is not included in the article's Creative Commons license and your intended use is not permitted by statutory regulation or exceeds the permitted use, you will need to obtain permission directly from the copyright holder. To view a copy of this license, visit http://creativecommons. org/licenses/by/4.0/.

\section{References}

1. Ley TJ, Miller C, Ding L, Raphael Bj, Mungall AJ, Robertson A, et al. Genomic and epigenomic landscapes of adult de novo acute myeloid leukemia. N Engl J Med 2013;368:2059-74.

2. Falini B, Mecucci C, Tiacci E, Alcalay M, Rosati R, Pasqualucci $\mathrm{L}$, et al. Cytoplasmic nucleophosmin in acute myelogenous leukemia with a normal karyotype. N Engl J Med. 2005;352:254-66.

3. Sportoletti P, Grisendi S, Majid SM, Cheng K, Clohessy JG, Viale A, et al. Npm1 is a haploinsufficient suppressor of myeloid and lymphoid malignancies in the mouse. Blood. 2008;111:3859-62.

4. Khanna-Gupta A, Abayasekara N, Levine M, Sun H, Virgilio M, $\mathrm{Nia} \mathrm{N}$, et al. Up-regulation of translation eukaryotic initiation factor $4 \mathrm{E}$ in nucleophosmin 1 haploinsufficient cells results in changes in CCAAT enhancer-binding protein alpha activity: implications in myelodysplastic syndrome and acute myeloid leukemia. J Biol Chem. 2012;287:32728-37.

5. Gale RE, Green C, Allen C, Mead AJ, Burnett AK, Hills RK, et al. The impact of FLT3 internal tandem duplication mutant level, number, size and interaction with NPM1 mutations in a large cohort of young adult patients with acute myeloid leukemia. Blood. 2008;111:2776-84.

6. Adolfsson J, Mansson R, Buza-Vidas N, Hultquist A, Liuba K, Jensen CT, et al. Identification of Flt3+ lympho-myeloid stem cells lacking erythro-megakaryocytic potential a revised road map for adult blood lineage commitment. Cell. 2005; 121:295-306.

7. Ferreira R, Ohneda K, Yamamoto M, Philipsen S. GATA1 function, a paradigm for transcription factors in hematopoiesis. Mol Cell Biol. 2005;25:1215-27.

8. Yoshida K, Toki T, Okuno Y, Kanezaki R, Shiraishi Y, Sato-Otsubo A, et al. The landscape of somatic mutations in Down syndrome-related myeloid disorders. Nat Genet. 2013;45:1293-9.

9. Ayala R, Martinez-Lopez J, Gilsanz F. Acute myeloid leukemia and transcription factors: role of erythroid Kruppel-like factor (EKLF). Cancer Cell Int. 2012;12:25.

10. Figueroa ME, Abdel-Wahab O, Lu C, Ward PS, Patel J, Shih A, et al. Leukemic IDH1 and IDH2 mutations result in a hypermethylation phenotype, disrupt TET2 function, and impair hematopoietic differentiation. Cancer Cell. 2010; 18:553-67.

11. Kats LM, Reschke M, Taulli R, Pozdnyakova O, Burgess K, Bhargava P, et al. Proto-oncogenic role of mutant IDH2 in leukemia initiation and maintenance. Cell Stem Cell. 2014;14:329-41.

12. Shih AH, Jiang Y, Meydan C, Shank K, Pandey S, Barreyro L, et al. Mutational cooperativity linked to combinatorial epigenetic gain of function in acute myeloid leukemia. Cancer Cell. 2015; 27:502-15.

13. Lindstrom MS. NPM1/B23: a multifunctional chaperone in ribosome biogenesis and chromatin remodeling. Biochem Res Int. 2011;2011:195209.

14. Falini B, Sportoletti P, Brunetti L, Martelli MP. Perspectives for therapeutic targeting of gene mutations in acute myeloid leukaemia with normal cytogenetics. $\mathrm{Br} \mathrm{J}$ Haematol. 2015;170:305-22.

15. Falini B, Brunetti L, Martelli MP. Dactinomycin in NPM1-mutated acute myeloid leukemia. N Engl J Med. 2015;373:1180-2. 